\title{
Gabapentin for the treatment of spasticity in patients with spinal cord injury
}

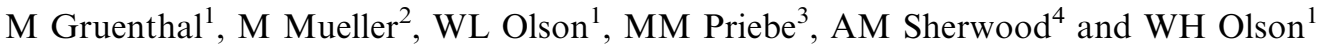 \\ ${ }^{1}$ Departments of Neurology and Internal Medicine, and ${ }^{2}$ Division of Physical Medicine and Rehabilitation, University \\ of Louisville School of Medicine, Louisville, KY; ${ }^{3}$ Department of Physical Medicine and Rehabilitation, Baylor \\ College of Medicine and Veterans Affairs Medical Center, Spinal Cord Injury Service, Houston, Texas; ${ }^{4}$ Division of \\ Restorative Neurology and Neurobiology, Baylor College of Medicine, Houston, Texas. Address reprint requests to \\ Dr Gruenthal at The University of Louisville Department of Neurology, HSC \#113, 500 S Preston Street, Louisville, \\ KY 40292, USA.
}

\begin{abstract}
Our serendipitous observations suggested that some patients with spasticity appeared to have improved following the administration of the anticonvulsant drug gabapentin. As some patients with spasticity are either refractory to or intolerant of established medical treatments, we conducted this study to investigate the effect of gabapentin on spasticity in patients with spinal cord injury. Twenty-five patients with spinal cord injury and spasticity received oral gabapentin (2400 $\mathrm{mg}$ over $48 \mathrm{~h}$ ) in a randomized, double blind, placebo-controlled crossover study. We assessed responses by measuring the Ashworth spasticity scale, muscle stretch reflexes, presence of clonus and reflex response to noxious stimuli. Patient ratings were obtained using a Likert Scale. Administration of gabapentin, but not placebo, was associated with an $11 \%$ reduction in spasticity as measured by the Ashworth Scale $(P=0.04)$ and by a $20 \%$ reduction in the Likert Scale $(P=0.0013)$. Significant changes were not obtained for the other measures. The data obtained suggest that gabapentin may be useful in the management of spasticity associated with spinal cord injury.
\end{abstract}

Keywords: spasticity; spinal cord injury; gabapentin

\section{Introduction}

Painful muscle spasms, spasticity and rigidity affect up to $67 \%$ of patients with a severe spinal cord injury. ${ }^{1}$ Painful spasms and increased tone limit rehabilitation and the quality of life by interfering with physical therapy, sitting, patient transfers and other activities of daily living. Ambulation may be compromised in patients with incomplete lesions due to pain, decreased range of motion, tone and clonus. Medical therapy for spasticity following spinal cord injury has traditionally involved orally administered baclofen, dantrolene, benzodiazepines and clonidine. ${ }^{2-4}$ Baclofen is generally regarded as the drug of choice for spasticity of spinal origin. Its efficacy has been demonstrated in placebo controlled trials. ${ }^{5}$ It is less of a sedative than diazepam, ${ }^{2}$ and causes less weakness than dantrolene. ${ }^{6}$ Despite its demonstrated benefits, $25-35 \%$ of patients do not respond to oral baclofen. ${ }^{7}$ Furthermore, dose limiting neurotoxic adverse effects such as somnolence, ataxia, dizziness and confusion have been noted. ${ }^{2}$ Tizanidine has demonstrated effectiveness in clinical trials, but is not currently approved for use in North America. ${ }^{8}$

Surgical treatments for spasticity of spinal origin include tendon lengthening, neurotomy, dorsal rhizot-

Correspondence: Dr Michael Gruenthal omy and longitudinal myelotomy. Implantation of pumps for the administration of intrathecal baclofen had emerged as an effective procedure. ${ }^{9,10}$ Advantages of this technique include effectiveness in the absence of dose-limiting adverse effects. Nevertheless, hypotension, weakness and respiratory depression have been reported in placebo-controlled trials. ${ }^{10,11}$ Complications include mechanical failure, accidental overdosage and infection. ${ }^{9-11}$

Thus, despite available therapies, a treatment combining the attributes of clinical efficacy, safety, tolerability and cost-effectiveness is not available for all patients with spasticity of spinal cord origin. Our anecdotal experience with gabapentin, a recently marketed anticonvulsant, led us to conduct a prospective, double-blind, placebo controlled crossover trial of adjunctive oral gabapentin in the treatment of spasticity of spinal cord origin.

\section{Methods}

\section{Patient population}

Men and women age 18 and older with spinal cord injury were eligible for participation if they had uncontrolled spasticity, were not pregnant, had no 
to baseline data obtained immediately prior to initiation of that treatment. To investigate possible differences in the scores as a function of treatment order, unpaired comparisons between patients assigned to receive placebo first and gabapentin first were made using the Mann-Whitney U test. All $P$ values are twotailed. Observed differences were considered statistically reliable if the probability of a type one error was less than five percent.

\section{Results}

Three women and 25 men were enrolled in the trial and randomly assigned to receive either placebo followed by gabapentin or gabapentin followed by placebo. Three men withdrew after receiving placebo as the initial treatment and were not included in the analyses. No patients withdrew from the study during or after exposure to gabapentin, and no adverse events occurred. The mean age of the 25 patients completing the study was 42.5 years (range 21 to 60 years). The mean duration of spinal cord injury was 7.45 years (range 1 month to 26 years). The number of patients taking other antispasticity medications at the time of enrollment and the daily doses of those medications are shown in Table 3 . Four of the 25 patients were taking no medications, 13 were taking one, five were taking two, and three were taking three medications. No changes in these medications occurred during the study period. During the baseline evaluation, no significant differences on any measures were found between patients who subsequently received placebo first and those who received gabapentin first. Similarly, treat-

Table 3 Patient medications*

\begin{tabular}{lcc}
\hline Medication & \# of patients & $\begin{array}{c}\text { Mean dose } \\
\text { (mg/day) and range }\end{array}$ \\
\hline Baclofen (all oral) & 15 & $100(10-160)$ \\
Diazepam & 11 & $24(5-45)$ \\
Dantrolene & 3 & $167(150-200)$ \\
Clonazepam & 2 & $1(0.5-1.5)$ \\
Clonidine & 1 & 0.05 \\
\hline
\end{tabular}

*The mean daily dose and range of antispasticity medications, as well as the number of the 25 study patients who were taking these medications prior to and during the study ment order did not affect the data obtained with administration of either placebo or gabapentin.

The median scores obtained for each measure are shown in Table 4. Administration of gabapentin resulted in an $11 \%$ reduction in the median Ashworth Scale score $(z=2.011, P=0.044)$ and a $20 \%$ reduction in the median Likert Scale score $(z=3.214, P=0.0013)$ when compared to placebo. Other measures did not yield significant differences. Similar results were obtained when responses to gabapentin were compared to baseline data. No significant changes in any measure were seen when responses to placebo were compared to baseline data. The small decrease in the Likert Scale score and increases in the Ashworth and clonus scores associated with placebo administration were not statistically significant.

\section{Discussion}

This prospective, double blind placebo controlled crossover study was designed to investigate the potential role of gabapentin in the treatment of spasticity following spinal cord injury. The results indicate that gabapentin administration for $48 \mathrm{~h}$ is associated with significant improvements in patient ratings of spasticity and in Ashworth Scale scores. Although the observed differences are small, many patients described substantial improvements in quality of life associated with taking gabapentin. These data indicate that gabapentin may be useful in the management of patients with spasticity associated with spinal cord injury. Previous studies in healthy volunteers and patients with epilepsy have shown gabapentin to be safe and well tolerated, and to have very favorable pharmacokinetic properties. $^{12}$

Several aspects of this study warrant further comment. The gabapentin dose was chosen after our impression, based on an uncontrolled observation, that $900 \mathrm{mg}$ daily in three divided doses was minimally effective. We chose an 11 day washout period because some patients with spasticity appeared to remain improved for up to ten days following a 2 day exposure, suggesting a pharmacodynamic half-life in excess of the 5 to $9 \mathrm{~h}$ elimination half-life. The design was such that measurements were made following the administration of $2400 \mathrm{~g}$ of gabapentin in divided doses over a $48 \mathrm{~h}$ period, sufficient time to achieve

Table 4 Median scores for each measure*

\begin{tabular}{lccccc}
\hline Min-Max & LS $(0-5)$ & Ashworth (10-50) & Clonus (0-6) & Reflexes $(0-24)$ & Noxious $(0-6)$ \\
\hline P. Baseline & 3 & 21 & 0 & 12 & 2 \\
G. Baseline & 3 & 22 & 0 & 12 & 2 \\
Placebo & 2.5 & 22 & 1 & 12 & 2 \\
Gabapentin & 2 & 19.5 & 0.32 & 11 & 2 \\
P value & 0.0013 & 0.044 & 0.45 & 0.8 \\
\hline
\end{tabular}

*Abbreviations: Min-Max- possible minimum and maximum scores for each scale, LS- Likert Scale score $(0=$ none, $5=$ worst $)$, Ashworth- Ashworth Scale score, Noxoius- response to noxious stimulation, P. Baseline and G. Baseline- Baseline data obtained prior to administration of Placebo and Gabapentin, respectively 
steady-state concentrations. ${ }^{13}$ Statements regarding long term efficacy and tolerability cannot be made on the basis of these data. While this study did not include a formal long term followup, many patients requested continued access to gabapentin. Several of these patients have received gabapentin from either the study investigators or another physician responsible for their care, in doses up to $3600 \mathrm{mg} /$ day in three divided doses. In some instances, this has permitted a reduction or elimination of other medications for spasticity. While encouraging, these observations are anecdotal. Thus, although gabapentin has been shown to be well tolerated and effective as an anticonvulsant when administered to patients with epilepsy for up to 4 years, ${ }^{14}$ confirmation of long-term benefits in spasticity will require additional investigation.

This study was not designed to evaluate gabapentin as monotherapy in the treatment of spasticity. Over $80 \%$ of the patients studied were taking at least one other antispasticity medication, to which they were at least partially refractory. Thus, the data reported here should only be taken as evidence supporting the potential use of gabapentin as adjunctive therapy in the management of spasticity associated with spinal cord injury. While the properties of gabapentin make pharmacokinetic drug interactions unlikely, an additive or synergistic effect is suggested by the anecdotal observation that addition of gabapentin permitted a reduction in the dose of other antispasticity drugs.

Gabapentin has been approved in the US as adjunctive therapy for partial seizures with or without secondary generalization. This compound (1-[aminomethyl] cyclohexane acetic acid) was synthesized in an attempt to create a gamma aminobutyric acid (GABA) analogue which would penetrate the blood brain barrier. While it readily penetrates the blood brain barrier, ${ }^{13}$ its mechanism of action, which remains unknown, does not involve a direct interaction with GABA receptors. Thus, the mechanism(s) by which gabapentin reduces spasticity following spinal cord injury are uncertain, and could involve actions in the spinal cord and/or a direct effect on skeletal muscle. Gabapentin has a discrete binding site in the brain ${ }^{15,16}$ and appears to increase brain GABA concentrations. ${ }^{17}$ To our knowledge, no studies of the potential effects of this compound on the spinal cord or motor unit have been described.

From a clinical perspective, gabapentin has many advantageous properties. It does not bind plasma proteins, is not metabolized and does not induce hepatic enzymes. Absorption is not affected by food or repeated administration. Elimination is almost exclusively via the renal route and drug clearance is therefore linearly correlated with creatinine clearance. ${ }^{13}$ The results obtained in the present study, combined with the very favorable pharmacokinetic properties and adverse effect profile of gabapentin, suggest that this compound could be of significant clinical utility in the management of spasticity associated with spinal cord injury.

\section{Acknowledgements}

The authors gratefully acknowledge the support of the Frazier Rehabilitation Center and Jewish Hospital in Louisville, KY for providing space, personnel, secretarial and pharmacy support for this study. We are indebted to Victor Wood, RN the clinical nurse coordinator. Gabapentin and placebo were provided by Parke-Davis.

\section{References}

1 Maynard FM, Karunas RS, Waring WP. Epidemiology of spasticity following traumatic spinal cord inury. Arch Phys Med Rehabil 1990; 71: 566-569.

2 Hattab JR. Review of european clinical trials with baclofen. In: Feldman RG, Young RR, Koella WP (eds.). Spasticity: Disrodered motor control. Chicago: Year Book Medical Publishers, 1979; 71-85.

3 Chyatte SB, Basmajian JV. Dantrolene Sodium: Long-term effects in severe spasticity. Arch Phys Med Rehabil 1973; 54: $311-315$.

4 Nance PW, Shears AH, Nance DM. Reflex changes induced by clonidine in spinal cord injured patients. Paraplegia 1989; 27: $296-301$

5 Levine IM, Jossmann PB, DeAngelis V. Lioresal, a new muscle relaxant in the treatment of spasticity - A double-blind quantitative evaluation. Dis Nerv Syst 1977; 38: $1011-1015$.

6 Joynt RL. Dantrolene sodium: Long-term effects in patients with muscle spasticity. Arch Phys Med Rehabil 1976; 57: 212-217.

7 Corston RN, Johnson F, Godwin-Austen RB. The assessment of drug treatment of spastic gait. J Neurol Neurosurg Psychiatry 1981; 44: $1035-1039$.

8 Nance PW et al. Efficacy and safety of tizanidine in the treatment of spasticity in patients with spinal cord injury. Neurology 1994; 44: S44-S52.

9 Penn RD et al. Intrathecal baclofen for severe spinal spasticity. $N$ Engl J Med 1989; 320: 1517 - 1521.

10 Coffey RJ et al. Intrathecal baclofen for intractable spasticity of spinal origin: results of a long-term multicenter study. $J$ Neurosurg 1993; 78: 26-32.

11 Loubser PG et al. Continuous infusion of intrathecal baclofen: Long-term effects on spasticity in spinal cord injury. Paraplegia 1991; 29: $48-64$.

12 McLean MJ. Gabapentin. Epilepsia 1995; 36: S73-S86.

13 McLean MJ. Clinical pharmacokinetics of gabapentin. Neurology 1994; S17-S22.

14 Handforth A, Treiman DM. Efficacy and tolerance of long-term, high-dose gabapentin: Additional observations. Epilepsia 1994; 35: $1032-1037$.

15 Hill DR, Suman-Chauhan N, Woodruff GW. Localization of $\left[{ }^{3} \mathrm{H}\right]$ gabapentin to a novel site in rat brain: autoradiographic studies. Eur J Pharmacol 1993; 244: 303-309.

16 Suman-Chauhan $\mathrm{N}$ et al. Characterisation of $\left[{ }^{3} \mathrm{H}\right]$ gabapentin binding to a novel site in rat brain: homogenate binding studies. Eur J Pharmacol 1993; 344: 293 - 301.

17 Löscher W, Honack D, Taylor CP. Gabapentin increases aminooxyacetic acid-induced GABA accumulation in several regions of rat brain. Neurosci Lett 1991; 128: 150-154. 